\title{
Sagittal Spinopelvic Alignment in Asymptomatic Iranian Adults Aged 18 to 40 Years
}

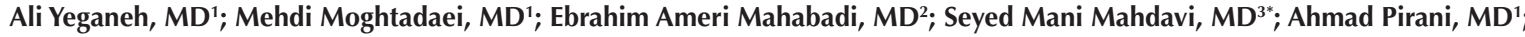 \\ Farshad Safdari, $\mathrm{PhD}^{4}$

\begin{abstract}
'Department of orthopedics, Rasoul-e-Akram Hospital, Iran University of Medical Sciences, Tehran, Iran ${ }^{2}$ Bone and Joint Reconstruction Research Center, Shafa Orthopedic Hospital, Iran University of Medical Sciences, Tehran, Iran ${ }^{3}$ Orthopedic Spine Surgery Department, Rasoul-e-Akram Hospital, Iran University of Medical Sciences, Tehran, Iran ${ }^{4}$ Department of Orthotics and Prosthetics, University of Social Welfare and Rehabilitation, Tehran, Iran
\end{abstract}

\begin{abstract}
Background: Recent studies have revealed the increasing importance of sagittal spinopelvic alignment. Knowing the values of sagittal spinopelvic parameters, which are affected by ethnicity, is essential in the normal asymptomatic population. In the current study, these parameters were measured in a sample of asymptomatic Iranian population.

Methods: Seventy asymptomatic participants without complaint of musculoskeletal problems were enrolled. They had no complaint about musculoskeletal problems. Lateral full-length spinal and pelvic $\mathrm{x}$-rays were taken. The following parameters were measured on $\mathrm{x}$-rays and presented as mean \pm standard deviation: pelvic incidence (PI), sacral slope (SS), pelvic tilt (PT), lumbar lordosis (LL), thoracic kyphosis (TK), lumbar tilt (LT) and thoracic tilt (TT).

Results: The population consisted of 37 males and 33 females aged $26.6 \pm 4.27$ years. The mean values of PI, SS, PT, LL, TK, LT, and TT were $44.5 \pm 10.1,35.4 \pm 6.7,9.1 \pm 7.9,41.9 \pm 14.7,28.8 \pm 8.3,11.9 \pm 7.4$ and $-7.5 \pm 5.7$ (median: -10 ; 5 th percentile: -14 ; 95th percentile: 4.4 ) degrees, respectively. The variables were similar between males and females except for LL which was significantly higher in females $(37.8 \pm 16.5$ versus $46.5 \pm 11 ; P=0.013)$. In addition, the linear regression model revealed age to be independently related with PI (beta $=0.344 ; P=0.004)$ and PT (beta $=0.366 ; P=0.002)$. PI $(r=0.344, P=0.004)$ and PT ( $r$ $=0.359, P=0.002$ ) were positively correlated with age. Additionally, PI was positively correlated to SS, PT and LL and negatively to TT.

Conclusion: These findings may be used as referential values for sagittal spinopelvic parameters in the Iranian population. The positive correlation of PI with age questions the constancy of PI throughout life. However, larger studies are required.

Keywords: Aging, Alignment, Pelvis, Sagittal plane, Spinopelvic alignment

Cite this article as: Yeganeh A, Moghtadaei M, Ameri Mahabadi E, Mahdavi SM, Pirani A, Safdari F. Sagittal spinopelvic alignment in asymptomatic Iranian adults aged 18 to 40 years. Arch Iran Med. 2020;23(6):391-396. doi: 10.34172/aim.2020.32.
\end{abstract}

Received: January 24, 2019, Accepted: January 12, 2020, ePublished: June 1, 2020

\section{Introduction}

Maintaining balance in a standing position is a very sophisticated operation and requires the coordination of multiple neuromuscular and musculoskeletal systems. Different parts of the musculoskeletal system, which is a complex of several biomechanical systems, should function properly on all three planes. Since the upright body is, in fact, an open kinematic chain, the balance should be evaluated in association with assessment of the alignment of all parts of the body, including the vertebral column, pelvis and lower extremities. ${ }^{1,2}$

In recent years, it has been found that in addition to the coronal alignment of the body, sagittal alignment is also very important in the individual's function and balance. $^{3}$ One of the most important findings in this regard was recognition of biomechanical relationship and significant correlation between the pelvis and the spine. ${ }^{4}$ Currently, it is obvious that the pelvic morphology (pelvic incidence) and its orientation (pelvic tilt) can significantly affect the geometry of the lumbar spine, especially lumbar morphology (lumbar lordosis). ${ }^{5,6}$ It has been shown in various studies that examining the sagittal balance and its preservation after surgery plays an important role in understanding and recognizing the pathomechanics of the spinal column as well as pain relief and functional improvement in several conditions, including spinal deformities in adults. ${ }^{7,8}$

Furthermore, abnormal sagittal alignment is associated with increased energy consumption to maintain good balance and effective posture, reduced movement ability, increased risk of falling, reduced strength of spinal extensors, and reduced quality of life. ${ }^{3,9-11}$

Considering the importance of global sagittal balance, various studies have been done to measure the sagittal spinopelvic parameters in normal and asymptomatic populations across different societies and races. ${ }^{4,5,12-14}$ Knowing these normal values can be helpful in examination of sagittal spinopelvic alignment, preoperative planning, 
choice of appropriate treating method, improvement of surgical outcomes and evaluating treatment outcomes in different age groups. ${ }^{15-17}$ In addition, the distinction between normal and abnormal spinal alignment in sagittal plane is much more difficult than the coronal plane, and knowledge of normal values seems necessary. ${ }^{18}$ The availability of reference values may be useful for investigating the variability of the parameters in different individuals and can be very helpful in understanding the underlying mechanisms of various spinal disorders. ${ }^{5,6,19,20}$

The importance of normal values of sagittal spinopelvic parameters necessitates measuring these values in different populations, including the Iranian population. To our knowledge, there has been no similar study in asymptomatic Iranian adults. In the current study, the sagittal spinopelvic parameters were measured in a sample of asymptomatic Iranian adults.

\section{Material and Methods}

Between September 2017 and August 2018, 107 consecutive asymptomatic subjects aged 18 to 40 were enrolled in the current study. These participants had no complaint about pain or other problems in spine and lower extremities. Prior to the study, the approval of the Ethics Committee was obtained and all of the volunteers signed a written consent. Exclusion criteria included generalized musculoskeletal diseases such as rheumatoid arthritis, any deformity or disorder of the spinal column (spondylolisthesis, pars interarticularis defects, etc) which was visible on $\mathrm{x}$-rays, scoliosis based on Adam's test, a history or clinical findings of pelvis or hip abnormality, Legg-Calve-Perthes disease, limb length discrepancy $>1$ $\mathrm{cm}$, and a history of spinal or pelvic surgery.

After the initial examination, lateral full-length spinal and pelvic $\mathrm{x}$-rays were taken for the eligible participants. The criteria for acceptable images were visibility of the C7 and both femoral heads. At the time of radiography, the hip and knee joints were fully extended. Both shoulders and elbows were flexed until knuckles were placed on the supraclavicular fossa. The feet were shoulder width apart.

The measured variables on $\mathrm{x}$-rays included pelvic incidence (PI), sacral slope (SS), pelvic tilt (PT), lumbar lordosis (LL), thoracic kyphosis (TK), pelvic tilt (PT) and lumbar tilt (LT). The measurements were performed as follows:

PI: The angle between the perpendicular line to the midpoint of the sacral plate and the line connecting this point and the hip axis. SS: The angle between the tangent line on the sacral plate and the horizontal line was measured. The angle made by the line connecting the hip axis and middle of the sacral plate and the plumb line was measured as PT. TK was defined as Cobb's angle between the superior endplate of $\mathrm{T} 5$ and the inferior endplate of T12. LL was measured as Cobb's angle between the superior endplate of L1 and the inferior endplate of L5. TT was the angle between the plumb line and the line connecting the anterosuperior corner of $\mathrm{T} 1$ and the anteroinferior corner of T12. LT: The angle between the plumb line and the line connecting the anterosuperior corner of L1 and the anteroinferior corner of L5.

It should be noted that anterior TT and LT and posterior TT and LT were recorded as positive and negative values, respectively. In addition, hip axis was considered as the center of the line connecting the center of the femoral heads. All of the measurements were performed by two orthopedic surgeons using the MicroDicom software. The intra- and inter-observer reliability of these two surgeons was determined $>0.8$ in a pilot study. The mean of two measurements was considered as the final value for each variable.

Statistical analysis was performed using SPSS statistical software version 15.0. To investigate the presence of normal distribution, Kolmogorov-Smirnov test was utilized. The variables with normal distribution were presented as mean \pm standard deviation. In addition, the $5^{\text {th }}$ and $95^{\text {th }}$ percentiles were presented for variables without normal distribution. Independent samples $t$ test was used to compare the angles between males and females. Pearson's correlation test was used to examine the correlation between age and radiographic measurement and the correlation between different radiographic angles. Furthermore, linear regression analysis was used to investigate the relationship between sagittal spinopelvic parameters and age as the independent variable. $P<0.05$ was considered significant.

\section{Results}

In total, 107 volunteers were examined during the study. Of them, 37 subjects were excluded and the study was completed with 70 subjects ( 37 males and 33 females). The subjects were aged $26.6 \pm 4.27$ years (range: 18 to 40 years). There was no significant difference between males and females in term of age $(27.5 \pm 5.6$ years in males and $25.6 \pm 3.2$ years in females; $P=0.088$ ). Table 1 shows the averaged values of measured radiographic variables. Furthermore, these parameters were compared between males and females (Table 1). As shown, except for LL, other variables were similar between the two genders. LL was significantly higher in females $(P=0.013)$. Although the differences in PI and TT were considerable, they did not reach the significance level based on $P$ values (Table 1). In Table 2, the results of Pearson's correlation test between age and sagittal spinopelvic parameters are presented showing that PI $(P=0.004, \mathrm{r}=0.344)$ and PT ( $\mathrm{r}=0.359, P=0.002)$ were positively correlated with age. The results of linear regression analysis are shown in Table 3. Regression analysis revealed that PI and PT were related independently with the age. However, based on the regression results, it seems that age can poorly predict these parameters. 
Table 1. Mean Values of Sagittal Spinopelvic Parameters in All Subjects and Comparison between Males and Females

\begin{tabular}{|c|c|c|c|c|}
\hline & Total $(n=70)$ & Males $(n=37)$ & Females $(n=33)$ & $P$ Value \\
\hline Sacral slope (degree) & $35.4 \pm 6.7$ (33.8 to 37$)$ & $34.6 \pm 6.9$ (32.3 to 36.9$)$ & $36.3 \pm 6.4$ (34.1 to 38.5$)$ & 0.273 \\
\hline Pelvic incidence (degree) & $44.5 \pm 10.1$ (42.5 to 46.5$)$ & $42.4 \pm 9.6$ (39.2 to 45.6$)$ & $47 \pm 10.3(43.4$ to 50.6$)$ & 0.06 \\
\hline Pelvic tilt (degree) & $9.1 \pm 7.9(7.2$ to 11$)$ & $7.8 \pm 8.4$ ( 5 to 10.6$)$ & $10.6 \pm 7.1(8.1$ to 13.1$)$ & 0.141 \\
\hline Lumbar lordosis (degree) & $41.9 \pm 14.7$ (38.4 to 45.4$)$ & $37.8 \pm 16.5(32.3$ to 43.3$)$ & $46.5 \pm 11$ (42.6 to 50.4 ) & $0.013^{*}$ \\
\hline Thoracic Kyphosis (degree) & $28.8 \pm 8.3$ (26.8 to 30.8 ) & $28.8 \pm 8(26.2$ to 31.4$)$ & $28.8 \pm 8.8$ (25.7 to 31.9 ) & 0.996 \\
\hline Lumbar tilt (degree) & $11.9 \pm 7.4(10.1$ to 13.6$)$ & $13.2 \pm 6.9(10.9$ to 15.5$)$ & $10.6 \pm 7.8(7.9$ to 13.3$)$ & 0.146 \\
\hline Thoracic tilt (degree) & $\begin{array}{l}-7.5 \pm 5.7 \text { [median }=-10 ; 5^{\text {th }} \text { percentile } \\
\left.\quad=-14 ; 95^{\text {th }} \text { percentile }=4.4\right]\end{array}$ & $-6.3 \pm 6.4(-8.4$ to -4.2$)$ & $\begin{array}{c}-8.7 \pm 4.4\left[\text { median }=-10 ; 5^{\text {th }} \text { percentile }=\right. \\
\left.-14.6 ; 95^{\text {th }} \text { percentile }=0.7\right]\end{array}$ & 0.068 \\
\hline
\end{tabular}

*Significant difference at level of 0.05 .

Degree, 95\% confidence interval for variables with normal distribution.

Table 2. Correlation Between Age and Sagittal Spinopelvic Parameters

\begin{tabular}{|c|c|c|c|c|c|c|c|c|}
\hline & Radiographic Parameter & Sacral Slope & Pelvic Incidence & Pelvic Tilt & Lumbar Lordosis & Thoracic Kyphosis & Lumbar Tilt & Thoracic Tilt \\
\hline \multirow{2}{*}{ Age } & Pearson's coefficient ${ }^{\circledR}$ & 0.09 & 0.344 & 0.359 & 0.23 & 0.197 & 0.148 & -0.119 \\
\hline & $P$ value & 0.458 & $0.004^{*}$ & $0.002 *$ & 0.055 & 0.102 & 0.222 & 0.325 \\
\hline
\end{tabular}

*Significant difference at level of 0.05 .

Table 3. Results of Linear Regression Analysis between Age (Independent Variable) and Sagittal Spinopelvic Parameters (Dependent Variables)

\begin{tabular}{|c|c|c|c|c|}
\hline & Adjusted R Square & B (Unstandardized) ( $95 \%$ Confidence Interval) & Beta (Standardized Coefficient) & $P$ Value \\
\hline Sacral slope & -0.006 & $0.128(-0.214$ to -0.469$)$ & 0.09 & 0.458 \\
\hline Pelvic incidence & 0.106 & 0.741 (0.252 to 1.229$)$ & 0.344 & $0.004 *$ \\
\hline Pelvic tilt & 0.121 & $0.613(0.236$ to 0.99$)$ & 0.366 & $0.002 *$ \\
\hline Lumbar lordosis & 0.039 & $0.72(-0.016$ to 1.455$)$ & 0.23 & 0.055 \\
\hline Thoracic kyphosis & 0.025 & $0.349(-0.071$ to 0.768$)$ & 0.197 & 0.102 \\
\hline Lumbar tilt & 0.007 & $0.232(-0.144$ to 0.607$)$ & 0.148 & 0.222 \\
\hline Thoracic tilt & 0 & $-0.144(-0.433$ to 0.146$)$ & -0.119 & 0.325 \\
\hline
\end{tabular}

*Significant $\mathrm{P}$ value.

The statistically significant correlations between sagittal spinopelvic parameters are shown in Table 4. As expected, PI was positively correlated with SS and PT. Further, PI had a positive correlation with LL. SS and PT, the sum of which equals PI, were also positively correlated to LL. Interestingly, PI and PT were negatively correlated to TT and not to TK. Finally, TK was positively correlated to TT and LT and not to LL.

\section{Discussion}

Investigating the anatomical, functional and biomechanical aspects of the vertebral column is always challenging. This complexity is due to the numerous factors and different structures, as well as the complex relationships between various spinal segments. Generally, appropriate relationship between spinal and pelvic parameters is necessary to maintain proper sagittal balance. Furthermore, it has been shown that attention to PI, other parameters of spinopelvic alignment and LL in preoperative surgical planning may result in improved surgical outcomes. ${ }^{21}$

Lack of knowledge about the values of these parameters in the Iranian population necessitated the current study. Since no similar study has been conducted in the Iranian community, the data of the current study, in spite of the limited sample size, may be useful as a basis for future studies.

The gender effect on the spinopelvic sagittal parameters remains controversial. ${ }^{4,22}$ In the current study, LL was significantly higher in females compared to males, but in other variables, there was no significant difference between the two genders. Interestingly, TK was approximately equal in the two genders. PI was considerably greater in females, but the difference did not reach a significant level. It seems that if more people had been examined, the difference between the two groups would have been significant. Similar to our findings, the difference between males and females in terms of LL was significant in the study by Vialle et al. However, in their study, contrary to ours, PI was significantly greater in females. ${ }^{5}$ Moon et al found that the mean values of PT and PI were significantly greater in females compared to males. ${ }^{22}$ Asai et al also found significant, but small differences between males and 
Table 4. Significant Correlations between Different Sagittal Spinopelvic and Spinal Parameters

\begin{tabular}{lcc}
\hline Couple of Variables & Pearson's Coefficient (R) & $\boldsymbol{P}$ Value \\
\hline PI \& SS & 0.628 & $<0.001$ \\
PI \& PT & 0.735 & $<0.001$ \\
PI \& LL & 0.602 & $<0.001$ \\
\hline PI \& TT & -0.275 & 0.021 \\
LL \& SS & 0.419 & $<0.001$ \\
LL \& PT & 0.418 & $<0.001$ \\
PT \& TT & -0.32 & 0.007 \\
TK \& TT & 0.263 & 0.028 \\
\hline TK \& LT & 0.506 & $<0.001$ \\
\hline
\end{tabular}

PI, pelvic incidence; SS, sacral slope; PT, pelvic tilt; LL, lumbar lordosis; TT, thoracic tilt; TK, thoracic kyphosis; LT, lumbar tilt

females in terms of TK, LL, PI and PT. ${ }^{4}$

Despite these findings, Janssen et al in France and Cho et al in Korea reported no difference between males and females in terms of LL, TK, and pelvic parameters. ${ }^{13,14}$ These controversial findings indicate that there is still a wide disagreement in the gender impact on spinopelvic parameters, and more studies are needed.

The mean PI in the current study was 44.5 degrees, which is close to the values obtained in the Chinese population (43.3 degrees and 44.6 degrees), but lower than that of the Caucasians (51.7 degrees) ${ }^{6,12,13,23}$ One of the highest PI values was reported in a study by Vialle et al in France on 300 asymptomatic individuals as 55 degrees. ${ }^{5}$ These findings may indicate the racial differences in the geometry of skeletal system that should be considered when evaluating and treating degenerative diseases and spinal deformities.

Based on the previous studies, normal TK and LL have a relatively wide range. The thoracic spine should have about 10 to 40 degrees of kyphosis and lumbar spine about 40 and 60 degrees of lordosis. In general, the lumbar spine curve should be mathematically about 30 degrees greater than the thoracic kyphosis. ${ }^{24}$ However, in the current study, such a ratio was not found for these two variables. TK and LL were 29 degrees and 42 degrees, respectively, which are mathematically only 13 degrees different, but each of the two curves alone was within the above-mentioned normal range. Furthermore, some studies have reported similar values for sagittal spinal curves. For example, Cho et al reported that TK and LL were approximately 29 and 48 degrees in a Korean population. ${ }^{13}$

Previous studies have demonstrated that PI is a constant anatomical parameter that is not affected by age, and represents the relationship between the sacrum and pelvis. The ability of the spine and pelvis to reach the sagittal balance depends on the PI and the changes of other spinopelvic parameters. ${ }^{25}$

In the current study, PI and PT were independently related to age. However, the correlation coefficients and regression coefficients were weak, indicating that the trend of the increase in these variables with aging probably occurs very slowly. Since PI was previously thought to be a relatively constant parameter over lifetime, this finding may indicate that the PI value also increases with age, although the change in PI may be small. This finding is confirmed by some other recent studies. Similar to the current study, Mac-Thiong et al stated that only PI and PT had a statistically significant correlation with age, in a way that PI and PT were greater in adults compared to children. ${ }^{14}$ Moon et al also confirmed the increase in PI with aging in both genders. ${ }^{22}$ On the contrary, however, Jentzsch et al found no relationship between age and PI. ${ }^{26}$

Vrtovec et al explained that the cause of increased PI with aging is an increase in the distance between the sacrum and femur leading to the increased PT and ultimately increased PI. ${ }^{27}$ Another interesting finding of the current study was that SS did not have a significant correlation with age. Since PI=PT+SS, and considering the very close correlation coefficients for PI $(r=0.359)$ and PT $(r=$ $0.344)$ with age, it is likely that the increase in PI at more advanced age is due to the increase in PT without decrease in SS. Moon et al also concluded that PT is probably the factor that affects PI. ${ }^{22}$

Contrary to our findings, in the study by Asai et al, SVA and TK increased and LL decreased with aging. ${ }^{4}$ Increased TK with aging can be due to the dehydration of intervertebral discs and, in some cases, osteoporotic fractures of the vertebrae. In some cases, however, the weakening of the spinal extensors has also been reported. ${ }^{28,29}$ In some other studies, LL reduction has been reported with aging. ${ }^{30,31} \mathrm{Zhu}$ et al reported that TK and PT had a significantly positive correlation with aging, while SS decreased with aging. ${ }^{12}$ Unlike the current study, the changes in SS and PT with aging in different directions have probably caused the consistency of PI value with aging.

As said earlier, the existence of several variables in investigating the sagittal spinopelvic alignment has made in difficult and challenging to evaluate the complex relationships between these variables and the effects of their changes on each other. In the current study, it was shown that there is a significantly positive correlation between PI as an anatomical parameter and SS and PT as positional parameters with considerably high coefficients. However, these two positional parameters were correlated with each other. These findings may confirm the findings of MacThiong et al demonstrating that the strong correlation coefficients of PI with PT and SS indicates the importance of pelvis morphology (PI) in determining and adjusting the direction and orientation of the sacrum (SS) as well as the pelvis (PT). ${ }^{14}$

Interestingly, similar to the current study, Mac-Thiong et al did not find a significant correlation between PT and SS (two variables indicating the orientation of the pelvis and sacrum) although these two variables are mathematically 
related. ${ }^{14}$ However, it is expected that since PI has a specific value, decreased SS could be associated with increased PT, and vice versa.

The relationship between the pelvis and lumbar parameters is one of the important issues in assessing the body position in the sagittal plane. In the current study, there was a significant correlation between PI and LL and there was a significant moderate correlation between LL and SS. In several studies, the relationship between lumbar spine morphology (LL) and pelvis morphology (PI), sacrum orientation (SS), or pelvis orientation (PT) has been well illustrated..$^{14,32,33}$ In contrast to the current study, Mac-Thiong et al and Berthonnaud et al found that LT, which shows the orientation of the lumbar spine, was also correlated with the orientation of the sacrum (SS). ${ }^{14,33}$

Another finding of the current study was the lack of a significant correlation between LL and TK. Theoretically, the two curves of LL and TK appear to change in response to each other to maintain a proper sagittal balance and posture. In addition, it seems that reducing LL with increasing TK due to spinal degeneration is the main cause of imbalance in the sagittal plane. ${ }^{13}$ It should be noted, however, that in the present study, lumbar orientation (LT) was correlated with thoracic morphology (TK). However, in some studies, the correlation between these two curves has been well shown. ${ }^{34}$ In the studies by Mac-Thiong et al and Berthonnaud et al, a significant correlation was found between TK and LL. ${ }^{14,33}$

Like all other studies, the current study also had limitations. The most important limitation of the study was the small sample size. In order to reliably measure the values of the sagittal spinal and spinopelvic parameters in the Iranian population and generalize the data, it is necessary to perform the study with many more participants. Moreover, we did not have the possibility of comparing these variables between different age groups in our study.

In conclusion, the findings of the current study provide useful information about the normal values of sagittal spinal and spinopelvic parameters in an Iranian population. The only significant difference in the sagittal spinal and spinopelvic parameters between the two genders was greater LL in females. The important finding of the current study was the increase in PI with aging, such that there was a positive and significant correlation between age and PI. In addition, PI was correlated with lumbar (LL) and thoracic (TK) morphology. Based on the findings of the present study, it seems that the change in PT with aging is probably the cause of PI changes with aging. More and larger studies are needed to obtain more reliable results.

\section{Authors' Contribution}

AY and EAM: Study concept and design. AY, EAM and SMM: Acquisition of data. SMM and FS: Analysis and interpretation of data. MM, SMM and FS: Drafting of the manuscript. MM and AP: Critical revision of the manuscript for important intellectual content. FS and AP: Statistical analysis. AP and EAM: Administrative, technical, and material support. AY and MM: Study supervision.

\section{Conflict of Interest Disclosures}

None.

\section{Ethical Statement}

This study was approved by the ethical committee of Iran University of Medical Sciences.

\section{References}

1. Lee CS, Park SJ, Chung SS, Lee KH. The effect of simulated knee flexion on sagittal spinal alignment: novel interpretation of spinopelvic alignment. Eur Spine J. 2013;22:1059-65. doi: 10.1007/s00586-013-2661-4.

2. Lazennec JY, Brusson A, Folinais D, Zhang A, Pour AE, Rousseau MA. Measuring extension of the lumbar-pelvicfemoral complex with the EOS(R) system. Eur J Orthop Surg Traumatol. 2015;25:1061-8. doi: 10.1007/s00590-015-16038.

3. Lenke LG. Sagittal balance. J Neurosurg Spine. 2014;20(5):51214.doi:10.3171/2013.10.SPINE13793.

4. Asai $\mathrm{Y}$, Tsutsui $\mathrm{S}$, Oka $\mathrm{H}$, Yoshimura $\mathrm{N}$, Hashizume $\mathrm{H}$, Yamada $\mathrm{H}$, et al. Sagittal spino-pelvic alignment in adults: The Wakayama Spine Study. PLoS One. 2017;12(6):e0178697. doi: 10.1371/journal.pone.0178697.

5. Vialle R, Levassor N, Rillardon L, Templier A, Skalli W, Guigui P. Radiographic analysis of the sagittal alignment and balance of the spine in asymptomatic subjects. J Bone Joint Surg Am. 2005;87(2):260-267. doi: 10.2106/JBJS.D.02043.

6. Vaz G, Roussouly P, Berthonnaud E, Dimnet J. Sagittal morphology and equilibrium of pelvis and spine. Eur Spine J. 2002;11:80-87. doi: 10.1007/s005860000224.

7. Labelle H, Roussouly P, Chopin D, Berthonnaud E, Hresko T, O'Brien M. Spino-pelvic alignment after surgical correction for developmental spondylolisthesis. Eur Spine J. 2008;17:11706. doi: 10.1007/s00586-008-0713-y.

8. Glassman SD, Berven S, Bridwell K, Horton W, Dimar JR. Correlation of radiographic parameters and clinical symptoms in adult scoliosis. Spine (Phila Pa 1976). 2005;30(6):682-8. doi: 10.1097/01.brs.0000155425.04536.f7.

9. Sinaki M, Brey RH, Hughes CA, Larson DR, Kaufman KR. Balance disorder and increased risk of falls in osteoporosis and kyphosis. Osteoporos Int. 2005;16:1004-1010. doi: 10.1007/s00198-004-1791-2.

10. Blechacz B, Gajic O. Images in clinical medicine. Severe kyphosis. N Engl J Med. 2008;358:e28. doi: 10.1056/ NEJMicm074057.

11. Stagnara P, De Mauroy JC, Dran G, Gonon GP, Costanzo G, Dimnet J, et al. Reciprocal angulation of vertebral bodies in a sagittal plane: approach to references for the evaluation of kyphosis and lordosis. Spine (Phila Pa 1976). 1982;7(4):33542. doi: 10.1097/00007632-198207000-00003.

12. Zhu Z, Xu L, Zhu F, Jiang L, Wang Z, Liu Z, et al. Sagittal alignment of spine and pelvis in asymptomatic adults: norms in Chinese populations. Spine (Phila Pa 1976). 2014;39(1):E16. doi: 10.1097/BRS.0000000000000022.

13. Cho Y. Evaluation of global sagittal balance in Koreans adults. Korean Neurosurg Soc. 2017;60(5):560-6. doi: 10.3340/ jkns.2016.1212.002.

14. Mac-Thiong JM, Labelle H, Berthonnaud E, Betz RR, Roussouly P. Sagittal spinopelvic balance in normal children and adolescents. Eur Spine J. 2007;16(2):227-34. doi: 10.1007/s00586-005-0013-8.

15. Le Huec JC, Leijssen P, Duarte M, Aunoble S. Thoracolumbar imbalance analysis for osteotomy planification using a new method: FBI technique. Eur Spine J. 2011;20(Suppl. 5):669_ 
80. doi: 10.1007/s00586-011-1935-y.

16. Kim HJ, Bridwell KH, Lenke LG, Park MS, Song KS, Piyaskulkaew C, et al. Patients with proximal junctional kyphosis requiring revision surgery have higher postoperative lumbar lordosis and larger sagittal balance corrections. Spine. 2014;39:E576-80. doi: 10.1097/BRS.0000000000000246.

17. Rose PS, Bridwell KH, Lenke LG, Cronen GA, Mulconrey DS, Buchowski JM, et al. Role of pelvic incidence, thoracic kyphosis, and patient factors on sagittal plane correction following pedicle subtraction osteotomy. Spine. 2009;34:78591. doi: 10.1097/BRS.0b013e31819d0c86.

18. Diebo BG, Henry J, Lafage V, Berjano P. Sagittal deformities of the spine: factors influencing the outcomes and complications. Eur Spine J. 2015;24(Suppl 1):S3-15. doi: 10.1007/s00586014-3653-8.

19. Jackson RP, Hales C. Congruent spinopelvic alignment on standing lateral radiographs of adult volunteers. Spine (Phila Pa 1976). 2000;25(21):2808-15. doi: 10.1097/00007632200011010-00014.

20. Labelle H, Roussouly P, Berthonnaud E, Dimnet J, O'Brien $\mathrm{M}$. The importance of spinopelvic balance in L5-S1 developmental spondylolisthesis: A review of pertinent radiologic measurements. Spine. 2005;30:S27-34. doi: 10.1097/01.brs.0000155560.92580.90.

21. Mehta VA, Amin A, Omeis I, Gokaslan ZL, Gottfried ON. Implications of spinopelvic alignment for the spine surgeon. Neurosurgery. 2012;70(3):707-21. doi: 10.1227/ NEU.0b013e31823262ea.

22. Moon JW, Shinn JK, Ryu D, Oh SY, Shim YS, Yoon SH. Pelvic incidence can be changed not only by age and sex, but also by posture used during imaging. Korean J Spine. 2017;14(3):7783. doi: 10.14245/kjs.2017.14.3.77.

23. Roussouly P, Gollogly S, Berthonnaud E, Dimnet J. Classification of the normal variation in the sagittal alignment of the human lumbar spine and pelvis in the standing position. Spine (Phila Pa 1976). 2005;30:346-53. doi: 10.1097/01. brs.0000152379.54463.65.

24. O'Brien MF, Lenke LG, Kuklo TR, Blanke KM. Spinal anatomy and alignment. In: O'Brien MF, Kuklo TR, Blanke KM, Lenke LG, eds. Radiographic Measurement Manual. USA: Scoliosis Research Society; 2008:1-10.

25. Boulay C, Tardieu C, Hecquet J, Benaim C, Mouilleseaux
B, Marty C, et al. Sagittal alignment of spine and pelvis regulated by pelvic incidence: standard values and prediction of lordosis. Eur Spine J. 2006;15(4):415-22. doi: 10.1007/ s00586-005-0984-5.

26. Jentzsch T, Geiger J, Bouaicha S, Slankamenac K, NguyenKim TD, Werner CM. Increased pelvic incidence may lead to arthritis and sagittal orientation of the facet joints at the lower lumbar spine. BMC Med Imaging. 2013;13:34. doi: 10.1186/1471-2342-13-34.

27. Vrtovec T, Janssen MM, Likar B, Castelein RM, Viergever MA, Pernuš F. Evaluation of pelvic morphology in the sagittal plane. Spine J. 2013;13:1500-9. doi: 10.1016/j.spinee.2013.06.034.

28. Sinaki M, McPhee MC, Hodgson SF, Merritt JM, Offord KP. Relationship between bone mineral density of spine and strength of back extensors in postmenopausal women. Mayo Clin Proc. 1986;61:116-22. doi: 10.1016/s00256196(12)65197-0.

29. Bartynski WS, Heller MT, Grahovac SZ, Rothfus WE, KursLasky M. Severe thoracic kyphosis in the older patient in the absence of vertebral fracture: association of extreme curve with age. Am J Neuroradiol. 2005;26:2077-85.

30. Gelb DE, Lenke LG, Bridwell KH, Blanke K, McEnery KW. An analysis of sagittal spinal alignment in 100 asymptomatic middle and older aged volunteers. Spine (Phila Pa 1976). 1995;20:1351-8.

31. Takemitsu $Y$, Harada $Y$, Iwahara $T$, Miyamoto $M$, Miyatake Y. Lumbar degenerative kyphosis. Clinical, radiological and epidemiological studies. Spine (Phila Pa 1976). 1988;13:131726.

32. Legaye J, Duval-Beaupère G, Hecquet J, Marty C. Pelvic incidence: a fundamental pelvic parameter for threedimensional regulation of spinal sagittal curves. Eur Spine J. 1998;7:99-103. doi: 10.1007/s005860050038.

33. Berthonnaud E', Dimnet J, Roussouly P, Labelle H. Analysis of the sagittal balance of the spine and pelvis using shape and orientation parameters. J Spinal Disord. 2005;18:40-7. doi: 10.1097/01.bsd.0000117542.88865.77.

34. Labelle H, Mac-Thiong JM, Roussouly P. Spino-pelvic sagittal balance of spondylolisthesis: a review and classification. Eur Spine J. 2011;20(Suppl 5):641-6. doi: 10.1007/s00586-0111932-1. 